\title{
A de novo molecular generation method using latent vector based generative adversarial network
}

\author{
Oleksii Prykhodko ${ }^{1,3+}$, Simon Viet Johansson ${ }^{1,3^{*}+}$ (D), Panagiotis-Christos Kotsias ${ }^{1}$, Josep Arús-Pous ${ }^{1,2}$, \\ Esben Jannik Bjerrum ${ }^{1}$, Ola Engkvist ${ }^{1}$ and Hongming Chen ${ }^{1,4^{*}}$
}

\begin{abstract}
Deep learning methods applied to drug discovery have been used to generate novel structures. In this study, we propose a new deep learning architecture, LatentGAN, which combines an autoencoder and a generative adversarial neural network for de novo molecular design. We applied the method in two scenarios: one to generate random drug-like compounds and another to generate target-biased compounds. Our results show that the method works well in both cases. Sampled compounds from the trained model can largely occupy the same chemical space as the training set and also generate a substantial fraction of novel compounds. Moreover, the drug-likeness score of compounds sampled from LatentGAN is also similar to that of the training set. Lastly, generated compounds differ from those obtained with a Recurrent Neural Network-based generative model approach, indicating that both methods can be used complementarily.
\end{abstract}

Keywords: Molecular design, Autoencoder networks, Generative adversarial networks, Deep learning

\section{Introduction}

There has been a surge of deep learning methods applied to cheminformatics in the last few years [1-5]. Whereas much impact has been demonstrated in deep learning methods that replace traditional machine learning (ML) approaches (e.g., QSAR modelling [6]), a more profound impact is the application of generative models in de novo drug design [7-9]. Historically, de novo design was performed by searching virtual libraries based on known chemical reactions alongside a set of available chemical building blocks [10] or by using transformational rules based on the expertise of medicinal chemists to design analogues to a query structure [11]. While many successes using these techniques have been reported in literature [12], it is worthwhile to point out that these

\footnotetext{
*Correspondence: Simon.johansson@astrazeneca.com; Hongming. chen71@hotmail.com

${ }^{\dagger}$ Oleksii Prykhodko and Simon Johansson contributed equally to this work

${ }^{1}$ Hit Discovery, Discovery Sciences, Biopharmaceutical R\&D, AstraZeneca, Gothenburg, Sweden

Full list of author information is available at the end of the article
}

methods rely heavily on predefined rules of structure generation and do not have the concept of learning prior knowledge on how drug-like molecules should be. In contrast, deep generative models learn how to generate molecules by generalizing the probability of the generation process of a large set of chemical structures (i.e., training set). Then, structure generation is basically a sampling process following the learned probability distribution $[7,8,13,14]$. It is a data-driven process and requires very few predefined rules.

Early attempted architectures were inspired by the deep learning methods used in natural language processing (NLP) [7, 15]. A recurrent neural network (RNN) trained with a set of molecules represented as SMILES strings [16] is able to generate a much bigger chemical space than the training set. Later on, the REINVENT method was proposed, which combines RNNs with reinforcement learning to generate structures with desirable properties [8]. Another architecture, the variational autoencoder (VAE), was also shown to generate novel chemical space $[9,17]$. This architecture is comprised of an encoder, that converts the molecule to a latent vector 
representation and a decoder, from which the latent representation tries to generate the input molecule again. By changing the internal latent representation and decoding it, new chemical space can be obtained. More studies followed that improved the architecture, in both making it more robust and improving the quality of the latent representation generated [18-20]. One special mention is the use of randomized SMILES [14, 21, 22]. Instead of using a unique SMILES representation for each molecule, different representations are used in every stage of the training. With this improvement, the quality of the chemical space generated in both RNNs and VAEs is much higher and the models tend to overfit much less. Besides the SMILES string based de novo structure generation methods, methods of generating molecules based on molecular graphs have also been proposed and, by using them, molecules can be directly generated step-bystep as molecular graphs [23-26].

Generative adversarial neural (GAN) networks [27] have become a very popular architecture for generating highly realistic content [28]. A GAN has two components, a generator and a discriminator, that compete against each other during training. The generator generates artificial data and the discriminator attempts to distinguish it from real data. The model is trained until the discriminator is unable to distinguish the artificial data from the real data. The first use in molecule generation was ORGAN [29] and its improved version, ORGANIC [30]. The former was tested with both molecular generation as well as musical scores, whereas the latter was targeted directly at inverse design of molecules. ORGANIC had trouble optimizing towards the discrete values from the Lipinski Rule of Five [31] heuristic score but showed some success in optimizing the QED [11] score. An algorithm combining GAN with RL was also used in RANC [32] and ATNC [33] where the central RNN was substituted by a differential neural computer (DNC) [34], a more advanced recurrent neural network architecture. The authors demonstrated that DNC-based architectures can handle longer SMILES and generate more diversity.

In this study, a new molecular generation strategy is described which combines an autoencoder and a GAN. The difference between this method and previous GAN methods such as ORGANIC and RANC is that the generator and discriminator network do not use SMILES strings as input, but instead $\mathrm{n}$-dimensional vectors derived from the code-layer of an autoencoder trained as a SMILES heteroencoder [35]. This allows the model to focus on optimizing the sampling and not worry about SMILES syntax issues. The decoder part of a pretrained heteroencoder [22] neural network was used to translate the generated $\mathrm{n}$-dimensional vector into molecular structures. We first trained the GAN on a set of ChEMBL [36] compounds and, after training, the GAN model was able to generate drug-like structures. Next, additional GAN models were trained on three target specific datasets (corresponding to EGFR, HTR1A and S1PR1 targets). Our results show that these GAN model can generate compounds which are similar to the ones in the training set but are still novel structures. We envision the LatentGAN to be a useful tool for de novo molecule design.

\section{Methods and materials}

\section{Heteroencoder architecture}

A heteroencoder is an autoencoder architecture trained on pairs of different representations of the same entity, i.e. different non-canonical SMILES of the same molecule. It consists of two neural networks, namely, the encoder and decoder, which are jointly trained as a transformation pipeline. The encoder is responsible for translating one-hot encoded SMILES strings into a numerical latent representation whereas the decoder accepts this latent representation and attempts to reconstruct one of the possible non-canonical SMILES string that it represents. The implementation followed the architecture previously reported in [22] with some changes (Fig. 1, bottom).

Initially, the one-hot encoded SMILES string is propagated through a two-layer bidirectional encoder with 512 Long Short-Term Memory [26] units per layer, half of which are used for the forward and half for the backward direction. The output of both directions is then concatenated and input to a feed-forward layer with 512 dimensions. As a regularizing step during training, the resulting vector is perturbed by applying additive zerocentered gaussian noise with a standard deviation of 0.1. The latent representation of the molecule is fed to a feedforward layer, the output of which is copied and inserted as hidden and cell states to a four-layer unidirectional LSTM RNN decoder with the same specifications as the encoder. Finally, the output of the last layer is processed by a feed-forward layer with softmax activation, to return the probability of sampling each character of the known character set of the dataset. Batch normalization with a momentum value of 0.9 [37] is applied on the output of every hidden layer, except for the gaussian noise layer.

The heteroencoder network was trained for 100 epochs with a batch size of 128 and using a constant learning rate of $10^{-3}$ for the first 50 epochs and an exponential decay following that, reaching a value of $10^{-6}$ in the final epoch. The decoder was trained using the teacher's forcing method [38]. The model was trained using the decoding loss function of categorial cross entropy between the decoded and the training SMILES. After training the heteroencoder, the noise layer is deactivated, resulting in a 


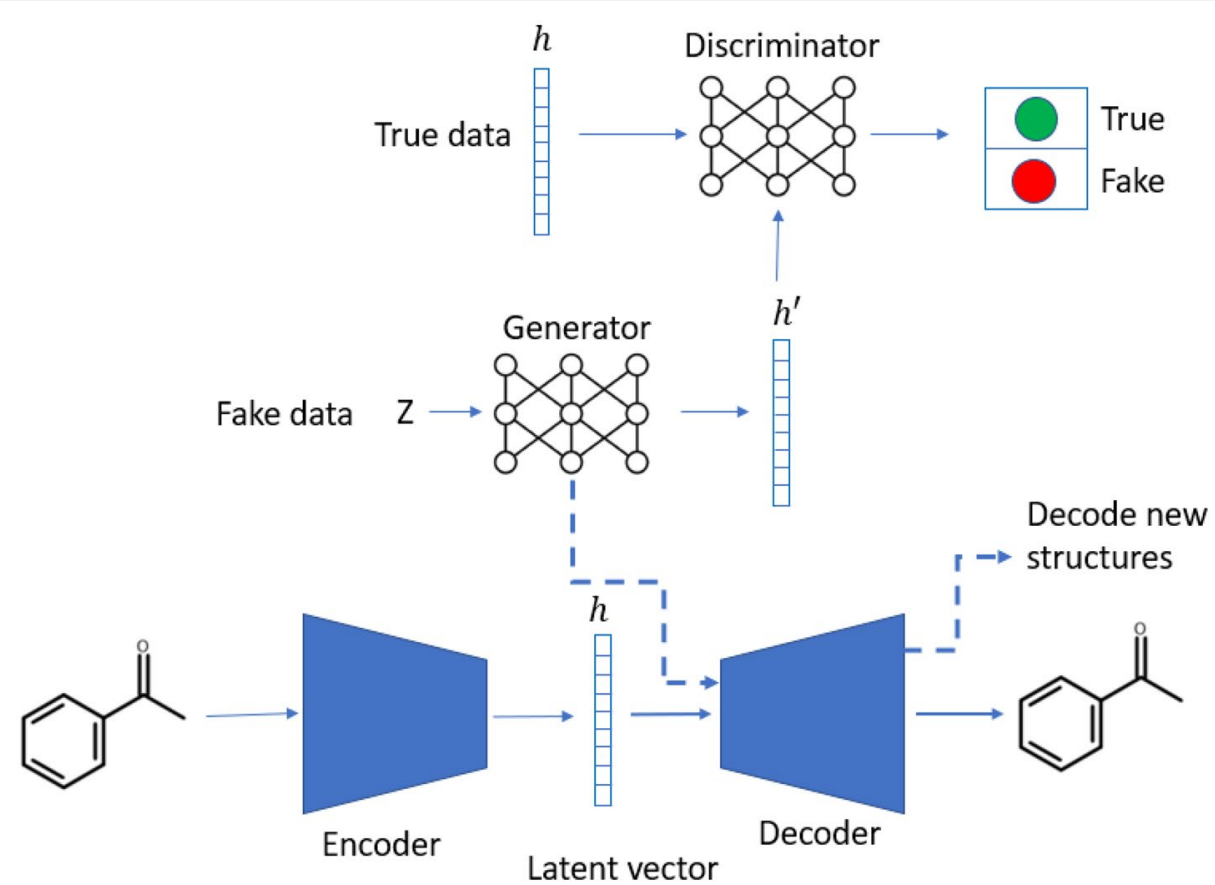

Fig. 1 Workflow of the LatentGAN. The latent vectors generated from the encoder part of the heteroencoder is used as the input for the GAN. Once the training of the GAN is finished, new compounds are generated by first sampling the generator network of the GAN and then converting the sampled latent vector into a molecular structure using the decoder component of the heteroencoder

deterministic encoding and decoding of the GAN training and sampled sets.

\section{The GAN architecture}

A Wasserstein GAN with gradient penalty (WGAN-GP) $[39,40]$ was chosen as a GAN model. Every GAN consists of two neural networks, generator and discriminator that train simultaneously (Fig. 1, top). First, the discriminator, usually called the critic in the context of WGANs, tries to distinguish between real data and fake data. It is formed by three feed-forward layers of 256 dimensions each with the leaky ReLU [41] activation function between, except for the last layer where no activation function was used. Second, the generator consists of five feed-forward layers of 256 dimensions each with batch normalization and leaky ReLU activation function between each.

\section{Workflow for training and sampling of the LatentGAN}

The heteroencoder model was first pre-trained on the ChEMBL database for mapping structures to latent vectors. To train the full GAN model, first the latent vector $h$ of the training set was generated using the encoder part of the heteroencoder. Then, it was used as the true data input for the discriminator, while a set of random vectors sampled from a uniform distribution were taken as fake data input to the generator. For every five batches of training for the discriminator, one batch was assigned to train the generator, so that the critic is kept ahead while providing the generator with higher gradients. Once the GAN training was finished, the Generator was sampled multiple times and the resulting latent vectors were fed into the decoder to obtain the SMILES strings of the underlying molecules.

\section{Dataset and machine learning models for scoring}

The heteroencoder was trained on 1,347,173 SMILES from the ChEMBL [36] dataset. This is a subset of ChEMBL 25 without duplicates that has been standardized using the MolVS [42] v0.1.1 package with respect to the fragment, charge, isotope, stereochemistry and tautomeric states. The set is limited to SMILES of containing only $[\mathrm{H}, \mathrm{C}, \mathrm{N}, \mathrm{O}, \mathrm{S}, \mathrm{Cl}, \mathrm{Br}]$ atoms and a total of 50 heavy atoms or less. Furthermore, molecules known to be active towards DRD2 were removed as part of an experiment for the heteroencoder (the process of which can be found at [35], which uses the same decoder model, but not the encoder). A set of randomly selected 100,000 ChEMBL compounds were later selected for training a general GAN model. Moreover, three target datasets (corresponding to EGFR, S1PR1 and HTR1A) were extracted from ExCAPE-DB [43] for training target specific GANs. The ExCAPE-DB datasets were then clustered into training and test sets so that chemical series were assigned either to the training or to the test set 
Table 1 Targeted data set and the performance of the SVM models

\begin{tabular}{lcrll}
\hline Target & Training set & Test set & \multicolumn{2}{l}{ SVM model } \\
\cline { 4 - 5 } & & & ROC-AUC & Kappa value \\
\hline EGFR & 2949 & 2326 & 0.850 & 0.56 \\
HTR1A & 48,283 & 23,048 & 0.993 & 0.90 \\
S1PR1 & 49,381 & 23,745 & 0.995 & 0.91 \\
\hline
\end{tabular}

Training set size (training set), test set size (test set), receiver operating characteristic area under the curve (ROC-AUC), kappa value

Table 2 The performance of heteroencoder in both the training and test sets

\begin{tabular}{lcll}
\hline Dataset & \# compounds & Validity (\%) & $\begin{array}{l}\text { Reconstruction } \\
\text { error (\%) }\end{array}$ \\
\hline Training set & 974,105 & 99 & 18 \\
Test set & 10,823 & 98 & 20 \\
\hline
\end{tabular}

Percent of valid SMILES strings generated by the decoder (validity), percent of molecules not reconstructed correctly from valid SMILES (reconstruction error)

(Table 1). To benchmark the performance of the targeted models, RNN based generative models for the three targets were also created by first training a prior RNN model on the same ChEMBL set used for training the heteroencoder model and then using transfer learning [7] on each focused target set. Target prediction models were calculated for each target using the Support vector machine learning (SVM) implementation in the Scikit-learn [44] package and the 2048-length FCFP6 fingerprint were calculated using RDKit [45].

\section{Related works}

A related architecture to the LatentGAN is the Adversarial Autoencoder (AAE) [46]. The AAE uses a discriminator to introduce adversarial training to the autoencoder and is trained typically using a 3-step training scheme of (a) discriminator, (b) encoder, (c) encoder and decoder, compared to the LatentGANs 2-step training. The AAE have been used in generative modeling of molecules to sample molecular fingerprints using additional encoder training steps [47], as well as SMILES representations [48, 49]. In other application areas, Conditional AAEs with similar training schemes have been applied to manipulate images of faces [50]. For the later application, approaches that have utilized multiple discriminators have been used to combine conditional VAEs and conditional GANs to enforce constraints on the latent space [51] and thus increase the realism of the images.

\section{Results and discussion}

\section{Training the heteroencoder}

The heteroencoder was trained on the 1,347,173 ChEMBL dataset compounds for 100 epochs. SMILES generated validity for the whole training set was $99 \%$ and $18 \%$ of the molecules were not reconstructed properly. Notice that the reconstruction error corresponds to decoding to a valid SMILES that belongs to a different

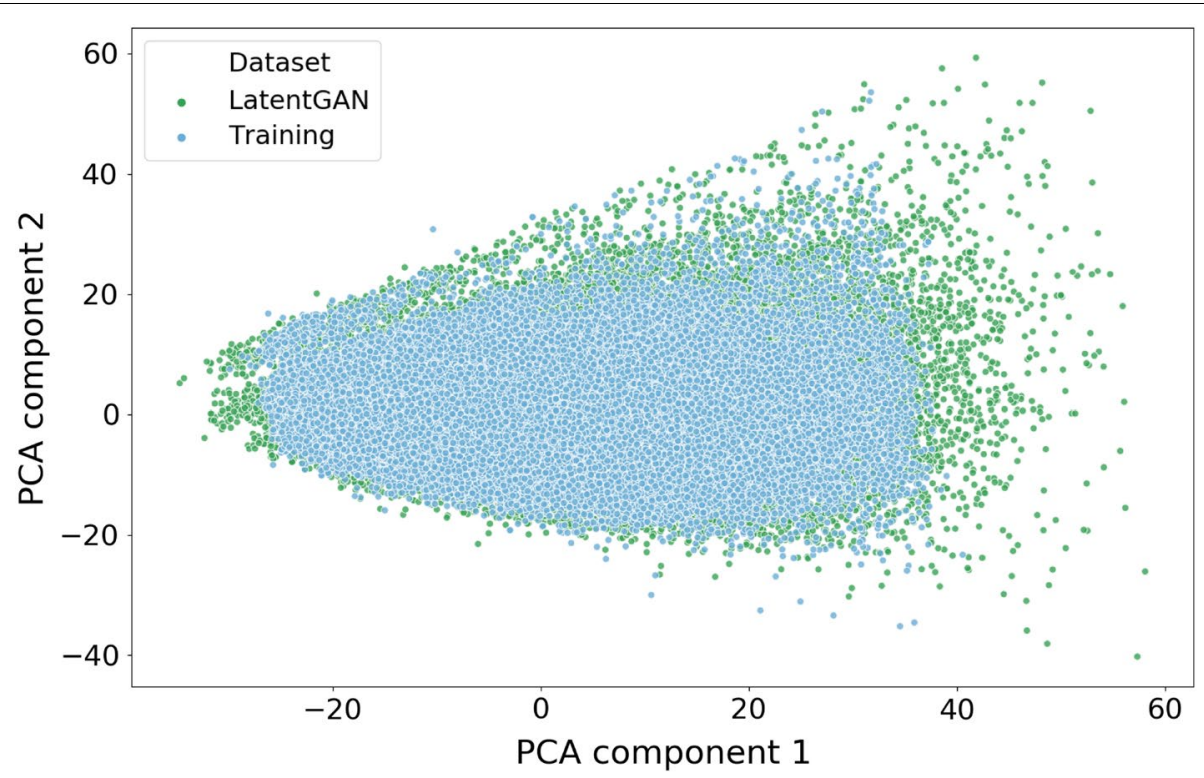

Fig. 2 Plot of the first two PCA components (explained variance 74.1\%) of a set of 200,000 generated molecules from the ChEMBL LatentGAN model using the MQN fingerprint 
Table 3 Metrics obtained from a 50,000 SMILES sample of all the models trained

\begin{tabular}{|c|c|c|c|c|c|c|c|}
\hline Dataset & Arch. & Valid (\%) & Unique (\%) & Novel (\%) & Active (\%) & $\begin{array}{l}\text { Recovered actives/total } \\
\text { actives (\%) }\end{array}$ & $\begin{array}{l}\text { Recovered } \\
\text { neighbors }\end{array}$ \\
\hline \multirow[t]{2}{*}{ EGFR } & GAN & 86 & 56 & 97 & 71 & 5.26 & 196 \\
\hline & RNN & 96 & 46 & 95 & 65 & 7.74 & 238 \\
\hline \multirow[t]{2}{*}{ HTR1A } & GAN & 86 & 66 & 95 & 71 & 5.05 & 284 \\
\hline & RNN & 96 & 50 & 90 & 81 & 7.28 & 384 \\
\hline \multirow[t]{2}{*}{ S1PR1 } & GAN & 89 & 31 & 98 & 44 & 0.93 & 24 \\
\hline & RNN & 97 & 35 & 97 & 65 & 3.72 & 43 \\
\hline
\end{tabular}

Dataset used (Dataset), Architecture used (Arch.), Percent of valid molecules in the sampled set (Valid), Percent of valid unique compounds (Unique), Percent of unique novel (not present in the training set) compounds (Novel), Percent of unique active compounds (Active), Recovered actives from the test set given the entire number of actives in the test set (Recovered actives/Total Actives), Recovered neighbors of active compounds using FCFP6 fingerprint with 2048 bits and a threshold Tanimoto similarity of 0.7

compound; reconstruction to a different SMILES of the same molecule is not counted as an error. Test set compounds were taken as input to the encoder and their latent values were calculated and then decoded to SMILES string, the validity and reconstruction error of test set are $98 \%$ and $20 \%$ respectively (Table 2).

\section{Training on the ChEMBL subset}

A LatentGAN was trained on a randomly selected 100,000 ChEMBL subset with the objective of obtaining drug-like compounds. The model was trained for 30,000 epochs until both discriminator and generator models had converged. Next, 200,000 compounds were generated from the LatentGAN model and were compared with the 100,000 ChEMBL training compounds to examine the coverage of the chemical space. The MQN [52] fingerprint was generated for all compounds in both sets and the top two principal components of a PCA were plotted (Fig. 2) and shows how both compound sets cover a similar chemical space.

\section{Training on the biased dataset}

Another interesting question to answer is if the LatentGAN can be trained to generate target specific compounds. The active compounds of training set were then used as the real data to train the LatentGAN. Each GAN model was trained 10,000 epochs and once the training was finished, 50,000 compounds were sampled from the generator and decoded with the heteroencoder. Then, three targets (EGFR, HTR1A and S1PR1) were selected and SVM target prediction models were built (see methods) to predict target activity on each target using the corresponding model (Table 3). Results show that in all cases validity was above $80 \%$ and the uniqueness of valid compound was $56 \%, 66 \%$ and $31 \%$ for EGFR, HTR1A and S1PR1 respectively. Comparing with the sample set of ChEMBL model these numbers are much lower, but this can be due to the smaller size of training sets.
Additionally, RNN models with transfer learning trained on the three targets (see "Methods and materials") show a higher percentage of validity, but their percentage of uniqueness is lower in all cases except for S1PR1. Regarding the novelty, the values are 97\%, 95\% and 98\% for EGFR, HTR1A and S1PR1 respectively and are slightly higher than the values of the RNN transfer learning models. This demonstrates that LatentGAN not only can generate valid SMILES but also most of them are novel to the training set, which is very important for de novo design tasks. All the sampled valid SMILES were then evaluated by the SVM models and a high percentage of the LatentGAN generated ones were predicted as active

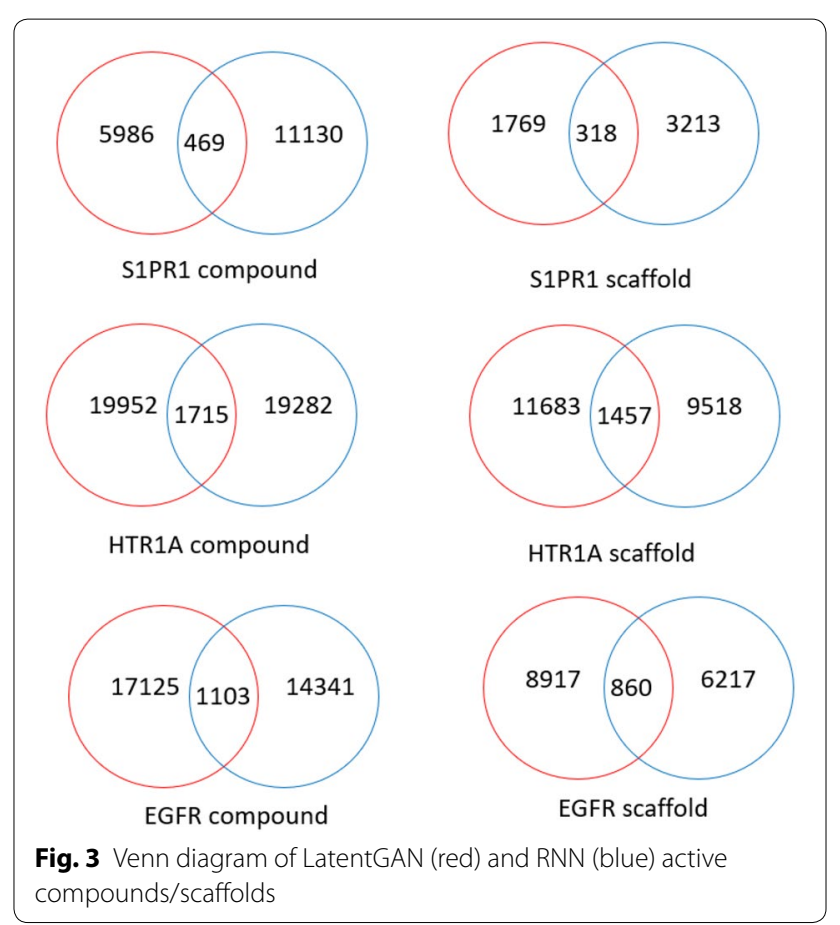




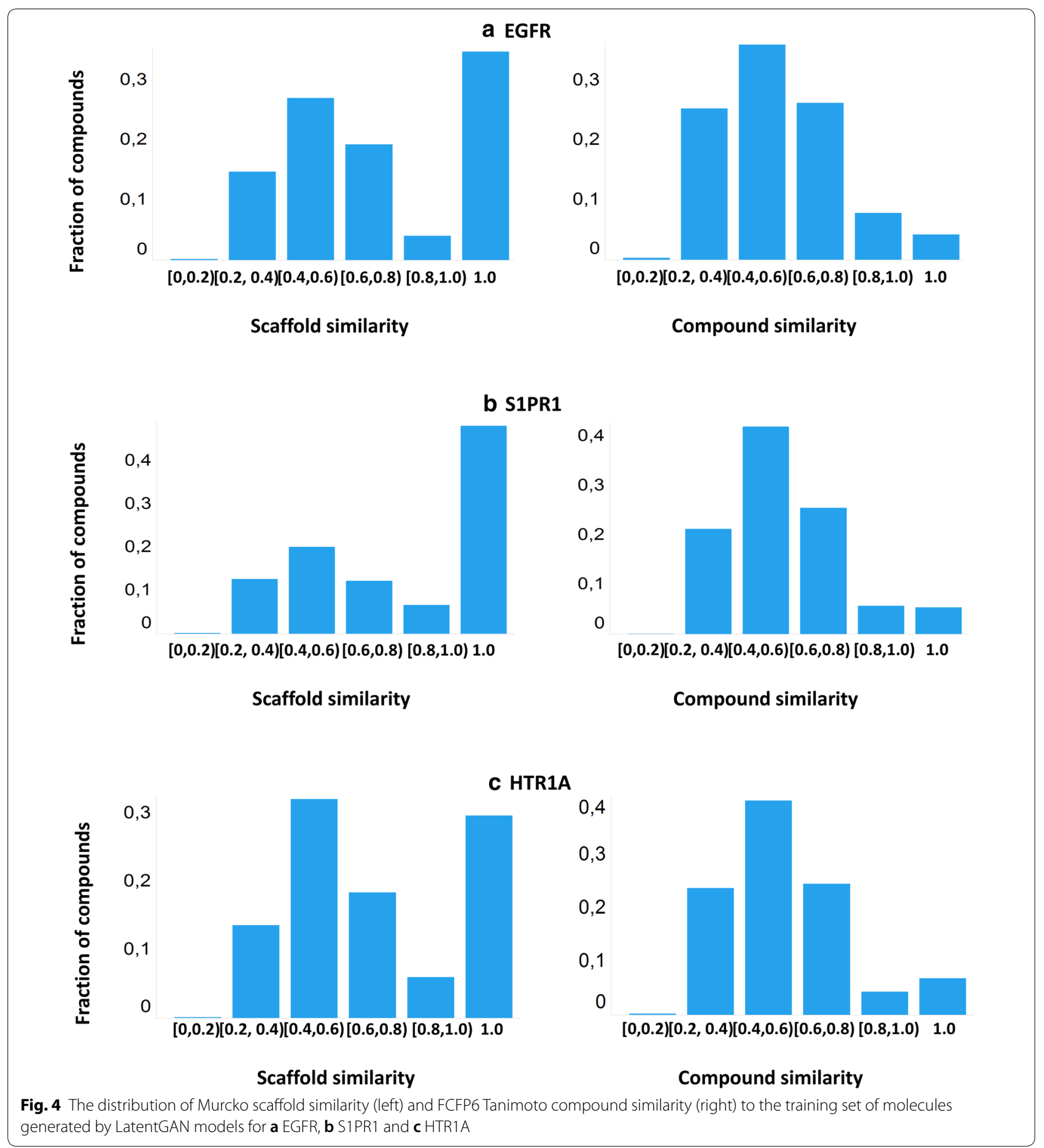

for these three targets $(71 \%, 71 \%$ and $44 \%$, for EGFR, HTR1A and S1PR1 respectively). These scores were better than the RNN models with respect to EGFR, but worse with respect to other two. Additionally, the comparison between LatentGAN and RNN generated active structures (Fig. 3) shows that the overlap is very small between the two architectures at both compound and scaffold levels. The compounds generated by LatentGAN were evaluated using the RNN model for a probabilistic estimation of whether the RNN model eventually would cover the LatentGAN output space, and it was shown to 
be very unlikely (see Additional file 1). This highlights that both architectures can work complementarily.

Full compound and Murcko scaffold [53] similarity was calculated between the actives in the sampled set and the actives in training set. Results (Fig. 4) show that, for each target, there are around $5 \%$ of generated compounds that are identical to the training sets. Additionally, there are around 25\%, 24\% and 21\% compounds having similarity lower than 0.4 to the training set in EGFR, HTR1A and S1PR1 respectively. This means that LatentGAN is able to generate very dissimilar compounds to the training set. In terms of scaffold similarity comparison, it is not surprising that the percentage of scaffolds identical to the training set is much higher for all the targets. Nevertheless, around 14\% of scaffolds in the sample set have low similarity to the training set $(<0.4)$ for all three tested cases.

A PCA analysis using the MQN fingerprint was performed to compare the chemical space of sampled sets and training sets of all targets and shows that the sampled compound sets cover most of the chemical space of the training sets (Fig. 5). Interestingly, there are some regions in the PCA plots where most of the sampled compounds around the training compounds are predicted as inactive, for example the left lower corner in EGFR (Fig. 5a) and the right-hand side region in S1PR1 (Fig. 5c). The training compounds in those regions are non-druglike compounds and outliers in the training set and the SVM models predicted them as inactive. No conclusive relationship between these regions of outliers and the scaffolds of lower similarity (Fig. 6). Additionally, we also evaluated the amount of the actives in the test set recovered by the sample set (Table 3). It is interesting to note that there are more active compounds belonging to the test set recovered by RNN model for all three targets, indicating that using multiple types of generative model for structure generation can be a viable strategy. Lastly, some examples generated by LatentGAN were drawn (Fig. 7) and the QED drug-likeness score [11] and Synthetic Accessibility (SA) score [54] distributions for each of the targets were plotted (Figs. 8 and 9, respectively). Training set compounds have a slightly higher drug-likeness, yet the
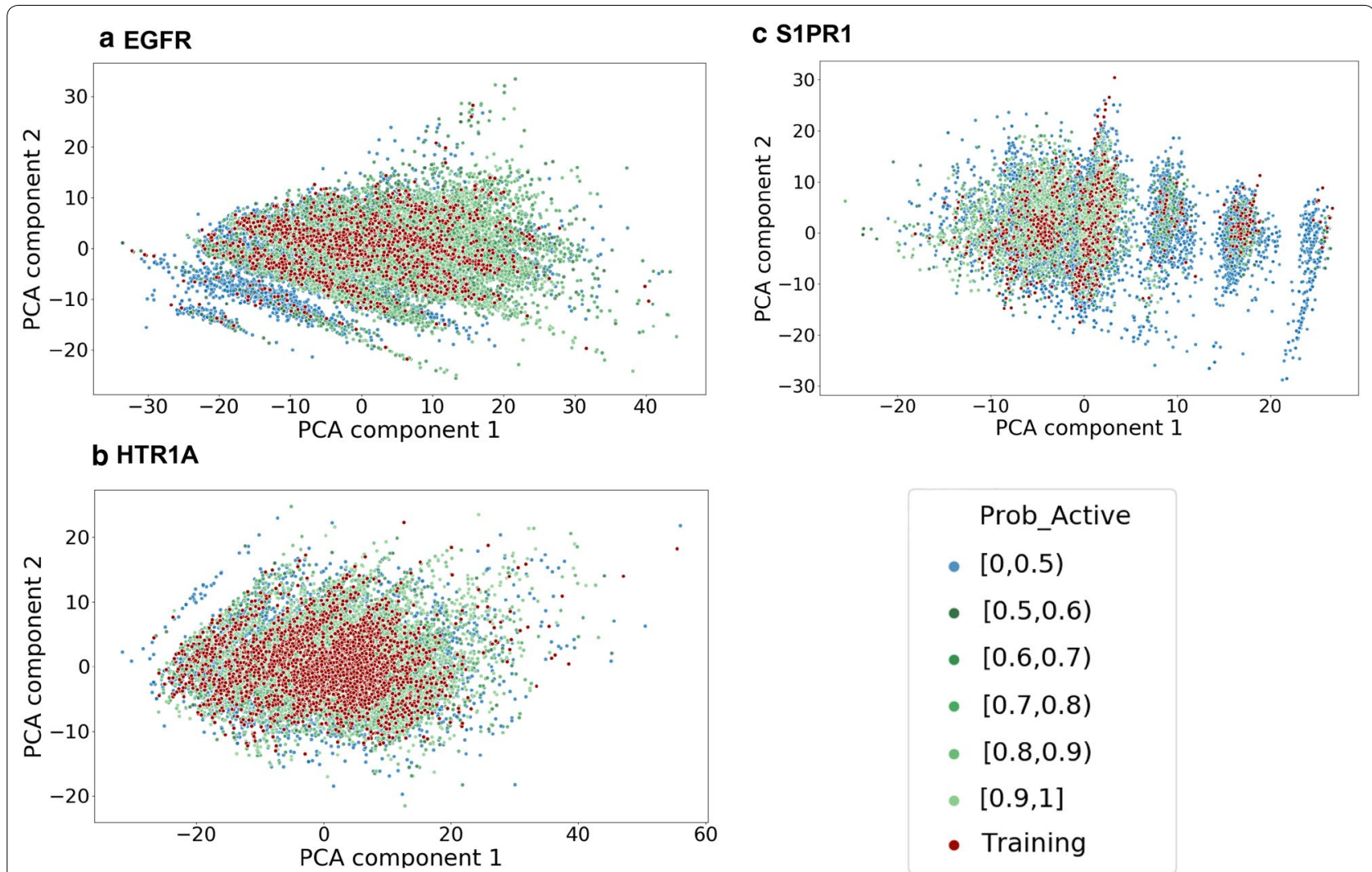

$$
\begin{aligned}
& \text { Prob_Active } \\
& \text { - }[0,0.5) \\
& \text { - }[0.5,0.6) \\
& \text { - }[0.6,0.7) \\
& \text { - }[0.7,0.8) \\
& \text { - }[0.8,0.9) \\
& \text { - }[0.9,1] \\
& \text { - Training }
\end{aligned}
$$

Fig. 5 PCA analysis for a EGFR (explained variance 82.8\%), b HTR1A (explained variance 75.0\%) and c S1PR1 (explained variance 79.3\%) dataset. The red dots are the training set, the blue dots are the predicted inactive compounds in the sampled set and other dots are the predicted actives in the sampled set with different level of probability of being active 
overall distributions are similar, showing that LatentGAN models can generate drug-like compounds.

\section{Comparison with similar generative networks}

The LatentGAN was assessed using the MOSES benchmark platform [48], where several generative metrics are used to evaluate the properties of molecular generative networks on a sample of 30,000 SMILES after training on a canonical SMILES subset of the ZINC database [55] of size 1,584,663. The full table of results for the MOSES benchmark is maintained and regularly updated at [56]. When compared to the similar structured networks of VAE, JTN-VAE [20] and AAE, it is noticeable that VAE model have an output distribution that has a significant overlap with the training set, as shown by the high scores of most test metrics (where the test set has a similar distribution to the training set) and the low novelty, indicating a mode collapse. When compared against the JTN-VAE and AAE models, the LatentGAN has shows comparable or better results in the Fréchet ChemNet Distance (FCD) [57], Fragment (Frag) and Scaffold (Scaf) similarities, while producing slightly worse results in the cosine similarity to the nearest neighbor in the test set (SNN).

\section{On the properties of autoencoder latent spaces}

In earlier VAE or AAE based architectures for generative molecular models, the role of the encoder is to forcefully fit the latent space of the training data to a Gaussian prior [47] or at least some continuous distribution [9], achieved in the latter with a loss function based on Kullback-Leibler (KL) divergence [58]. This requires the assumption
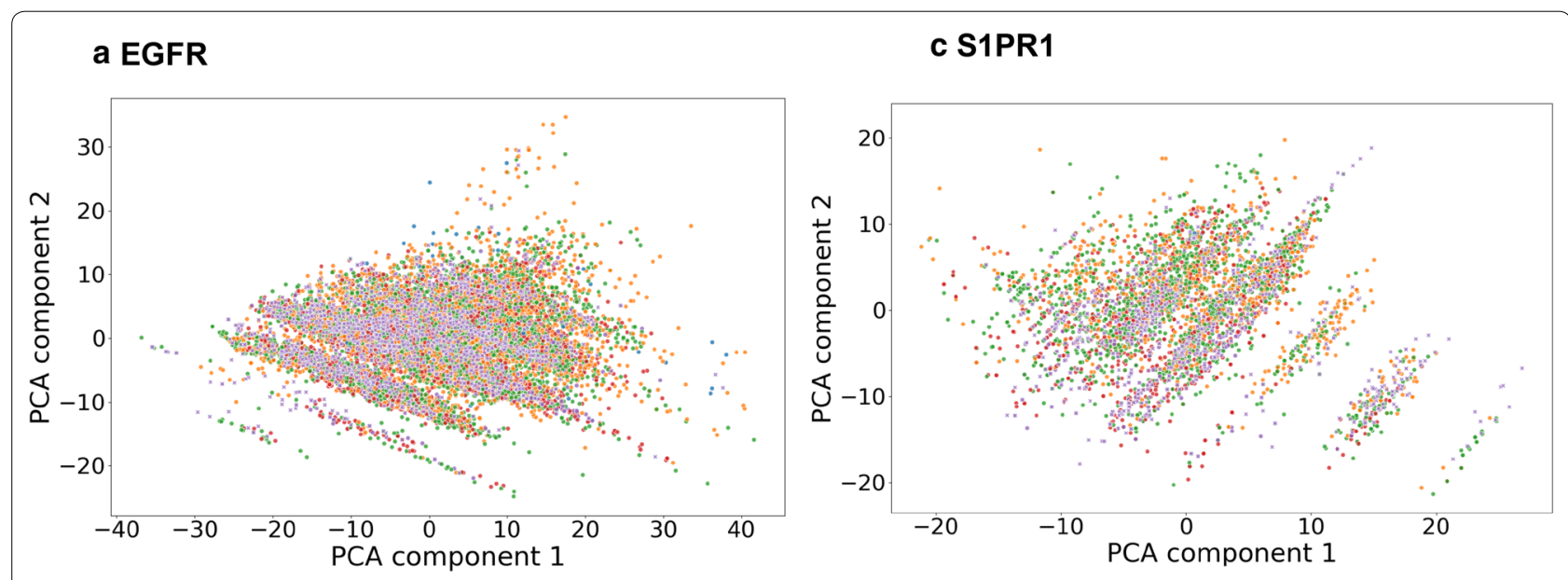

\section{b HTR1A}
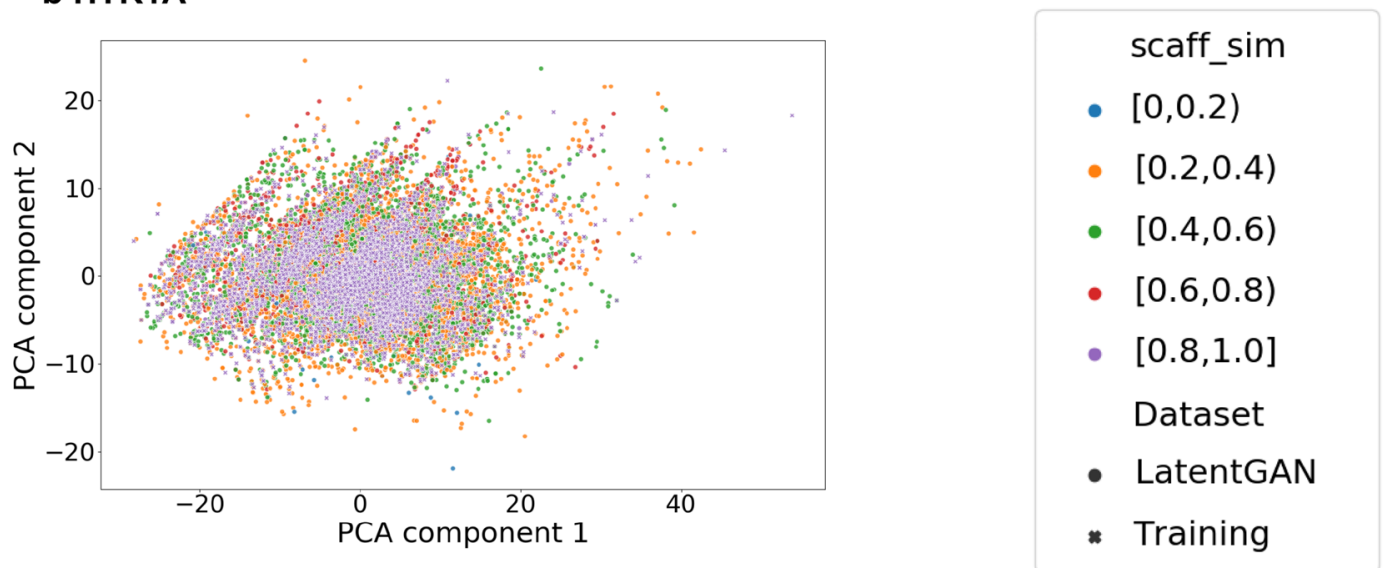

Fig. 6 The same PCA analysis, showing the Murcko scaffold similarities of the predicted active compounds for a EGFR (explained variance $80.2 \%$ ), $\mathbf{b}$ HTR1A (explained variance 74.1\%) and c S1PR1 (explained variance 71.3\%). Note that due to the lower amount in the outlier region of $\mathbf{c}$, the image has been rotated slightly. No significant relationship between the scaffold similarities and the regions was found. For a separation of the generated points by similarity interval, see Additional file 1 


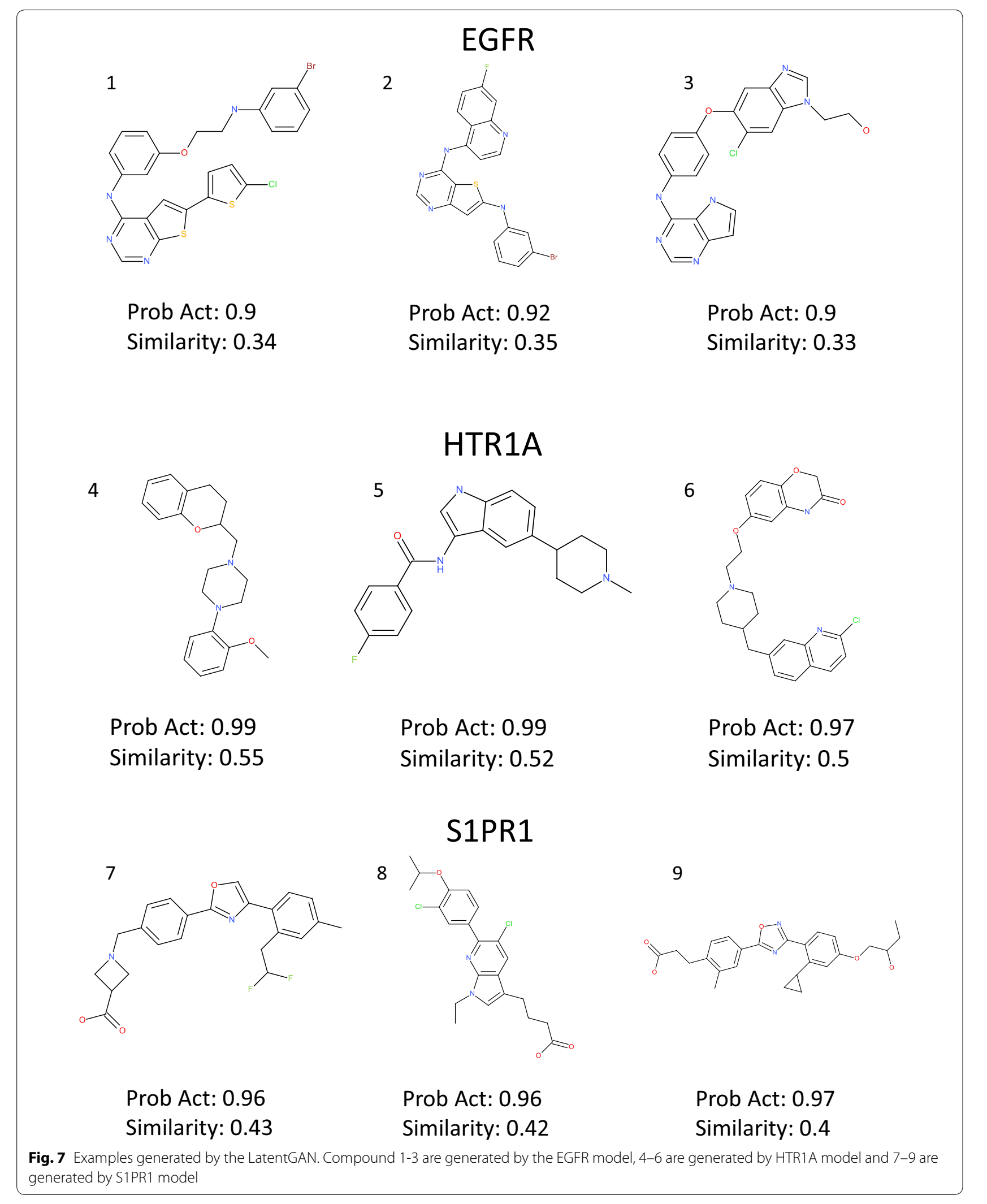


that by interpolating in the latent space between two molecules, the decoded molecule would then have either a structure or property that also lies between these molecules. This is not an intuitive representation, as the chemical space is clearly discontinuous-there is nothing between e.g. $\mathrm{C}_{4} \mathrm{H}_{10}$ and $\mathrm{C}_{5} \mathrm{H}_{12}$. The LatentGAN heteroencoder instead makes no assumption with regards to the latent space as no ground truth exists for this representation. Instead it is trained based strictly on the categorial cross entropy loss of the reconstruction. The result in a space of encoded latent vectors that the GAN later trains on that does not necessarily have to be continuous.

The complexity of the SMILES representation can also be a problem the training, as molecules of similar structures can have very different canonical SMILES when the starting atom changes, resulting in dissimilar latent representations of the same molecule. By training on non-canonical (random) SMILES [14, 21], this issue is alleviated since different non-canonical forms of the same molecule are encoded to the same latent space point which furthermore leads to a more chemically relevant latent space [22]. In addition, the multiple representations of the same molecule during training reduces the risk of overfitting the conditional probabilities of the decoder towards compounds who share a common substring of the SMILES in the canonical representation.

\section{Conclusions}

A new molecule de novo design method, LatentGAN, was proposed by combining a heteroencoder and a generative adversarial network. In our method, the pretrained autoencoder was used to map the molecular structure to latent vector and the GAN was trained using latent vectors as input as well as output, all in separate steps. Once the training of the GAN was finished, the sampled latent vectors were mapped back to structures by the decoder of the autoencoder neural network. As a first experiment, after training on a subset of ChEMBL compounds, the LatentGAN was able to generate similar drug-like compounds. We later applied the method on three target biased datasets (EGFR, HTR1A and S1PR1) to investigate the capability of the LatentGAN to generate biased compounds. Encouragingly, our results show that most of the sampled compounds from the trained model are predicted to be active to the target which it was trained against, with a substantial portion of the sampled compounds being novel with respect to the training set. Additionally, after comparing the structures generated from the LatentGAN and the RNN based models for the

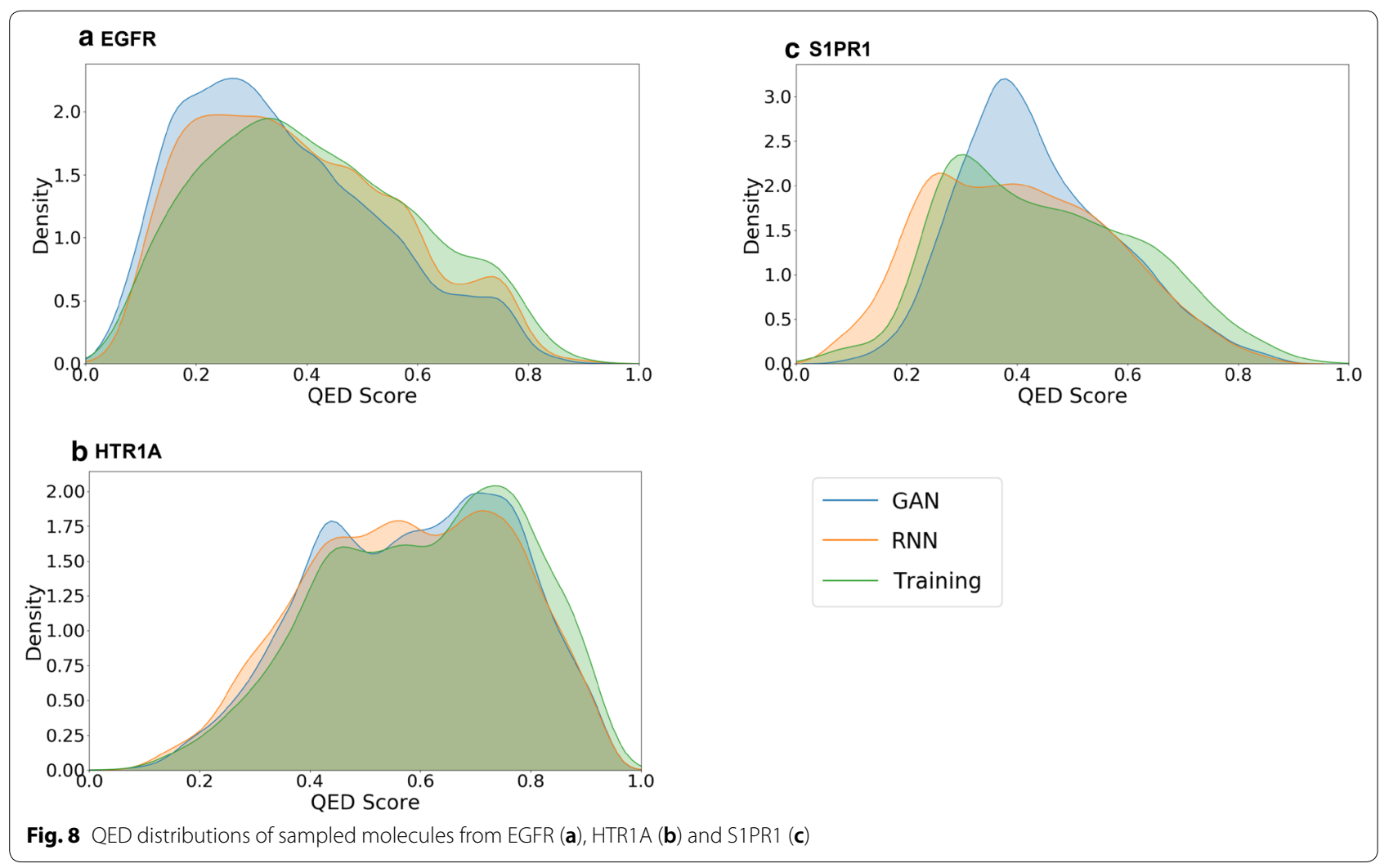


a EGFR

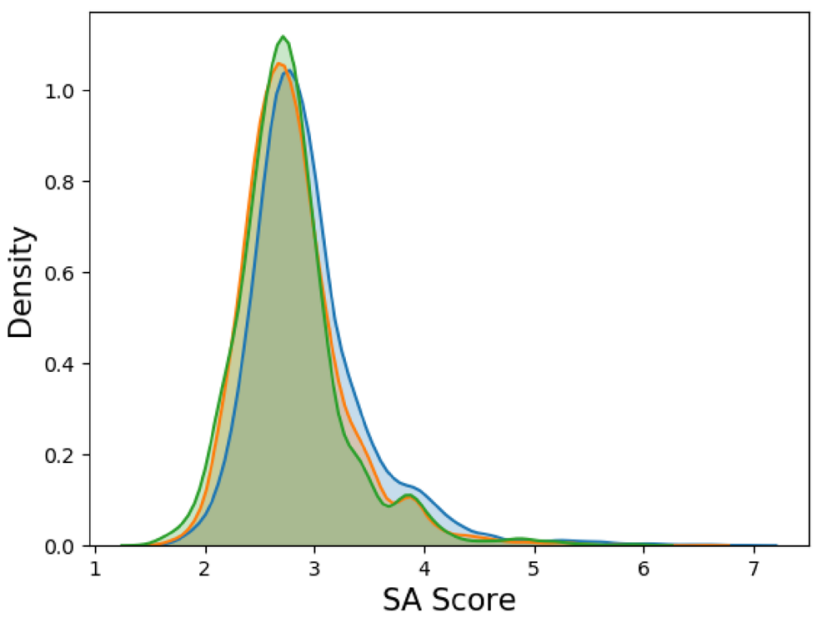

b HTR1A

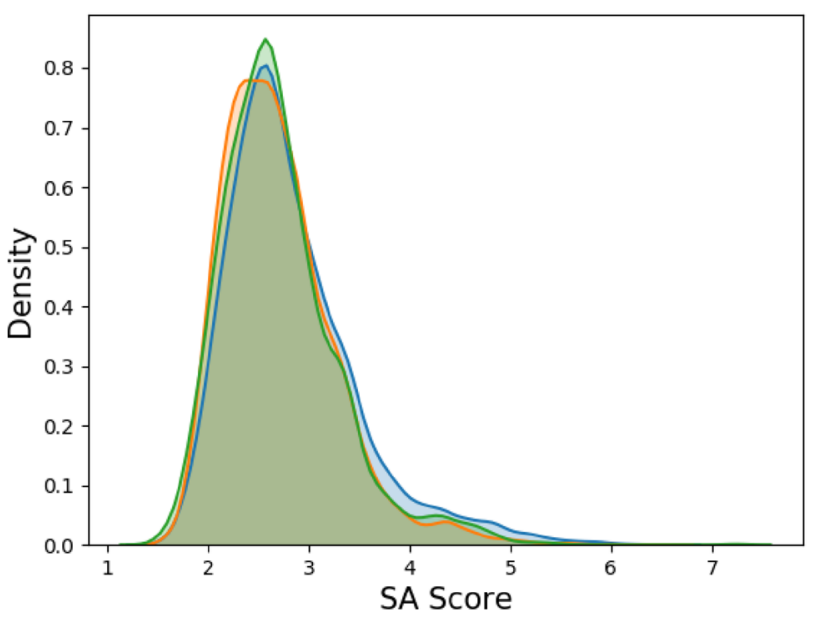

c S1PR1

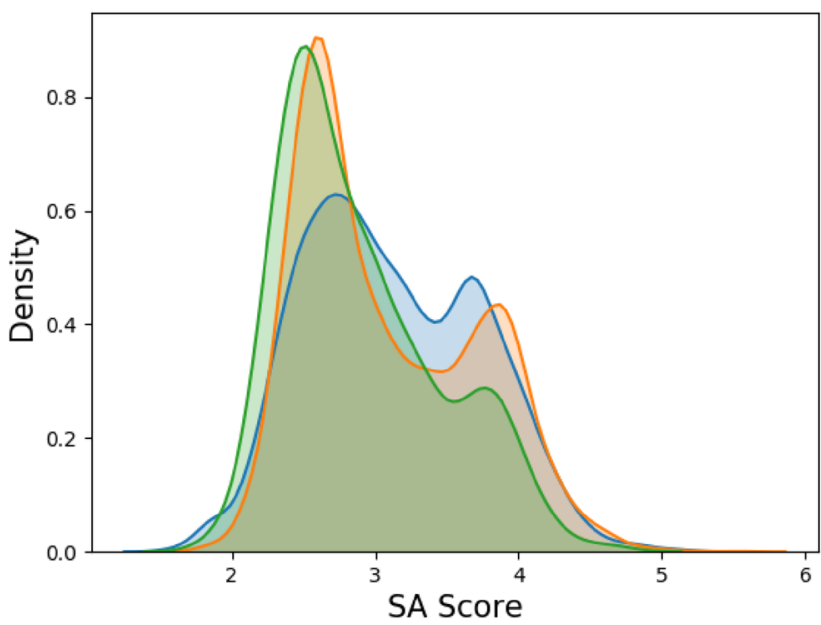

GAN

RNN

Training

Fig. 9 SA distributions of sampled molecules from EGFR (a), HTR1A (b) and S1PR1 (c)

corresponding targets, it seems that there is very little overlap among the two sets implying that the two types of models can be complementary to each other. In summary, these results show that LatentGAN can be a valuable tool for de novo drug design.

\section{Supplementary information}

Supplementary information accompanies this paper at https://doi. org/10.1186/s13321-019-0397-9.

Additional file 1. Supplementary figures and table.

\section{Acknowledgements}

Not applicable.

\section{Authors' contributions}

OP and SJ planned and performed the research. OP implemented the Wasserstein GAN and conducted training of the models. EJB and PK developed the heteroencoder. SJ, JAP and HC performed the analysis. SJ performed the benchmarking. OP, SJ, JAP and HC wrote the manuscript. OE and HC COsupervised the project. All authors read and approved the final manuscript.

\section{Funding}

Josep Arús-Pous is supported financially by the European Union's Horizon 2020 research and innovation program under the Marie Skłodowska-Curie Grant Agreement No. 676434, "Big Data in Chemistry" ("BIGCHEM," http://bigch em.eu).

\section{Availability of data}

The training sets and the trained heteroencoder model version used is available in through a GitHub repository (https://github.com/Dierme/latent-gan), which also contains the source code of the LatentGAN.

\section{Competing interests}

The authors declare that they have no competing interests. 


\begin{abstract}
Author details
1 Hit Discovery, Discovery Sciences, Biopharmaceutical R\&D, AstraZeneca, Gothenburg, Sweden. ${ }^{2}$ Department of Chemistry and Biochemistry, University of Bern, Bern, Switzerland. ${ }^{3}$ Department of Computer Science and Engineering, Chalmers University of Technology, Gothenburg, Sweden. ${ }^{4}$ Chemistry and Chemical Biology Centre, Guangzhou Regenerative Medicine and HealthGuangdong Laboratory, Science Park, Guangzhou, China.
\end{abstract}

Received: 13 September 2019 Accepted: 23 November 2019 Published online: 03 December 2019

\section{References}

1. Chen H, Engkvist O, Wang Y, Olivecrona M, Blaschke T (2018) The rise of deep learning in drug discovery. Drug Discov Today 23(6):1241-1250

2. Chen $\mathrm{H}$, Kogej T, Engkvist $\mathrm{O}$ (2018) Cheminformatics in drug discovery, an industrial perspective. Mol Inform 37(9-10):1800041

3. Ekins S (2016) The next era: deep learning in pharmaceutical research. Pharm Res 33(11):2594-2603

4. Gawehn E, Hiss JA, Schneider G (2016) Deep learning in drug discovery. Mol Inform 35(1):3-14

5. Hessler G, Baringhaus K-H (2018) Artificial intelligence in drug design. Molecules 23(10):2520

6. Lo Y-C, Rensi SE, Torng W, Altman RB (2018) Machine learning in chemoinformatics and drug discovery. Drug Discov Today 23(8):1538-1546

7. Segler MHS, Kogej T, Tyrchan C, Waller MP (2018) Generating focused molecule libraries for drug discovery with recurrent neural networks. ACS Cent Sci 4(1):120-131

8. Olivecrona M, Blaschke T, Engkvist O, Chen H (2017) Molecular de-novo design through deep reinforcement learning. J Cheminform. 9(1):48

9. Gómez-Bombarelli R, Wei JN, Duvenaud D, Hernández-Lobato JM, Sánchez-Lengeling B, Sheberla D, Aguilera-Iparraguirre J, Hirzel TD, Adams RP, Aspuru-Guzik A (2018) Automatic chemical design using a data-driven continuous representation of molecules. ACS Cent Sci 4(2):268-276

10. Schneider G, Geppert T, Hartenfeller M, Reisen F, Klenner A, Reutlinger M, Hähnke V, Hiss JA, Zettl H, Keppner S, Spänkuch B, Schneider P (2011) Reaction-driven de novo design, synthesis and testing of potential type II kinase inhibitors. Future Med Chem 3(4):415-424

11. Bickerton GR, Paolini GV, Besnard J, Muresan S, Hopkins AL (2012) Quantifying the chemical beauty of drugs. Nat Chem 4:90

12. Schneider P, Schneider G (2016) De novo design at the edge of chaos. J Med Chem 59(9):4077-4086

13. Arús-Pous J, Blaschke T, Ulander S, Reymond J-L, Chen H, Engkvist $O$ (2019) Exploring the GDB-13 chemical space using deep generative models. J Cheminform 11(1):20

14. Arús-Pous J, Johansson S, Prykhodko O, Bjerrum EJ, Tyrchan C, Reymond $\mathrm{J}$-L, Chen H, Engkvist O (2019) Randomized SMILES strings improve the quality of molecular generative models. J Cheminform 11:71. https://doi. org/10.1186/s13321-019-0393-0

15. Voss C (2015) Modeling molecules with recurrent neural networks. https ://csvoss.com/modeling-molecules-with-rnns. Accessed 12 Nov 2019

16. Weininger D (1988) SMILES, a chemical language and information system: 1: introduction to methodology and encoding rules. J Chem Inf Comput Sci 28(1):31-36

17. Blaschke T, Olivecrona M, Engkvist O, Bajorath J, Chen H (2018) Application of generative autoencoder in de novo molecular design. Mol Inform. https://doi.org/10.1002/minf.201700123

18. Lim J, Ryu S, Kim JW, Kim WY (2018) Molecular generative model based on conditional variational autoencoder for de novo molecular design. J Cheminform 10(1):31

19. Kusner MJ, Paige B, Hernández-Lobato JM (2017) Grammar variational autoencoder

20. Jin W, Barzilay R, Jaakkola T (2018) Junction tree variational autoencoder for molecular graph generation

21. Bjerrum EJ (2017) SMILES enumeration as data augmentation for neural network modeling of molecules

22. Bjerrum E, Sattarov B (2018) Improving chemical autoencoder latent space and molecular de novo generation diversity with heteroencoders. Biomolecules 8(4):131
23. Li Y, Vinyals O, Dyer C, Pascanu R, Battaglia P (2018) Learning deep generative models of graphs. Iclr, pp 1-16

24. Li Y, Zhang L, Liu Z (2018) Multi-objective de novo drug design with conditional graph generative model. J Cheminform 10(1):1-24

25. You J, Liu B, Ying R, Pande V, Leskovec J (2018) Graph convolutional policy network for goal-directed molecular graph generation

26. De Cao N, Kipf T (2018) MolGAN: an implicit generative model for small molecular graphs

27. Goodfellow I, Pouget-Abadie J, Mirza M, Xu B, Warde-Farley D, Ozair S, Courville A, Bengio Y (2014) Generative adversarial nets. In: NIPS

28. Karras T, Aila T, Laine S, Lehtinen J (2017) Progressive growing of GANs for improved quality, stability, and variation

29. Guimaraes GL, Sanchez-Lengeling B, Outeiral C, Farias PLC, Aspuru-Guzik A (2017) Objective-reinforced generative adversarial networks (ORGAN) for sequence generation models

30. Sanchez-Lengeling B, Outeiral C, Guimaraes GL, Aspuru-Guzik A (2017) Optimizing distributions over molecular space. An objective-reinforced generative adversarial network for inverse-design chemistry (ORGANIC)

31. Lipinski CA, Lombardo F, Dominy BW, Feeney PJ (2001) Experimental and computational approaches to estimate solubility and permeability in drug discovery and development settings 1PII of original article: S0169409X(96), 00423-1. The article was originally published in Advanced Drug Delivery Reviews 23 (1997). Adv Drug Deliv Rev 46(1-3):3-26

32. Putin E, Asadulaev A, Ivanenkov Y, Aladinskiy V, Sanchez-Lengeling B, Aspuru-Guzik A, Zhavoronkov A (2018) Reinforced adversarial neural computer for de novo molecular design. J Chem Inf Model 58(6):1194-1204

33. Putin $\mathrm{E}$, Asadulaev A, Vanhaelen $\mathrm{Q}$, Ivanenkov $Y$, Aladinskaya AV, Aliper A, Zhavoronkov A (2018) Adversarial threshold neural computer for molecular de novo design. Mol Pharm 15(10):4386-4397

34. Graves A, Wayne G, Reynolds M, Harley T, Danihelka I, Grabska-Barwińska A, Colmenarejo SG, Grefenstette E, Ramalho T, Agapiou J, Badia AP, Hermann KM, Zwols Y, Ostrovski G, Cain A, King H, Summerfield C, Blunsom P, Kavukcuoglu K, Hassabis D (2016) Hybrid computing using a neural network with dynamic external memory. Nature 538(7626):471-476

35. Kotsias P-C, Arús-Pous J, Chen H, Engkvist O, Tyrchan C, Bjerrum EJ (2019) Direct steering of de novo molecular generation using descriptor conditional recurrent neural networks (CRNNs)

36. Gaulton A, Hersey A, Nowotka ML, Patricia Bento A, Chambers J, Mendez D, Mutowo P, Atkinson F, Bellis LJ, Cibrian-Uhalte E, Davies M, Dedman N, Karlsson A, Magarinos MP, Overington JP, Papadatos G, Smit I, Leach AR (2017) The ChEMBL database in 2017. Nucleic Acids Res 45(D1):D945-D954

37. loffe S, Szegedy C (2015) Batch normalization: accelerating deep network training by reducing internal covariate shift

38. Williams RJ, Zipser D (2008) A learning algorithm for continually running fully recurrent neural networks. Neural Comput 1(2):270-280

39. Gulrajani I, Ahmed F, Arjovsky M, Dumoulin V, Courville A (2017) Improved training of wasserstein GANs

40. Luo Y (2018) EEG data augmentation for emotion recognition using a conditional wasserstein GAN. In: Proceedings of the annual international conference of the IEEE engineering in medicine and biology society, EMBS, pp 2535-2538

41. Maas AL, Hannun AY, Ng AY (2013) Rectifier nonlinearities improve neural network acoustic models

42. MolvS: molecule validation and standardization. (2019) https://molvs readthedocs.io/en/latest/. Accessed 13 Nov 2019

43. Sun J, Jeliazkova N, Chupakhin V, Golib-Dzib J-F, Engkvist O, Carlsson L, Wegner J, Ceulemans H, Georgiev I, Jeliazkov V, Kochev N, Ashby TJ, Chen H (2017) ExCAPE-DB: an integrated large scale dataset facilitating Big Data analysis in chemogenomics. J Cheminform 9(1):41

44. Pedregosa F, Varoquaux G, Gramfort A, Michel V, Thirion B, Grisel O, Blondel M, Prettenhofer P, Weiss R, Dubourg V, Vanderplas J, Passos A, Cournapeau D, Brucher M, Perrot M, Duchesnay É (2011) Scikit-learn: machine learning in python. J Mach Learn Res 12:2825-2830

45. Landrum G (2014) RDKit: open-source cheminformatics. http://www.rdkit org/. Accessed 2 Sept 2019

46. Makhzani A, Shlens J, Jaitly N, Goodfellow I, Frey B (2015) Adversarial autoencoders

47. Kadurin A, Aliper A, Kazennov A, Mamoshina P, Vanhaelen Q, Khrabrov K, Zhavoronkov A (2017) The cornucopia of meaningful leads: applying 
deep adversarial autoencoders for new molecule development in oncology. Oncotarget 8(7):10883-10890

48. Polykovskiy D, Zhebrak A, Sanchez-Lengeling B, Golovanov S, Tatanov O, Belyaev S, Kurbanov R, Artamonov A, Aladinskiy V, Veselov M, Kadurin A, Johansson S, Chen H, Nikolenko S, Aspuru-Guzik A, Zhavoronkov A (2018) Molecular sets (MOSES): a benchmarking platform for molecular generation models

49. Polykovskiy D, Zhebrak A, Vetrov D, Ivanenkov Y, Aladinskiy V, Mamoshina P, Bozdaganyan M, Aliper A, Zhavoronkov A, Kadurin A (2018) Entangled conditional adversarial autoencoder for de novo drug discovery. Mol Pharm 15(10):4398-4405

50. Zhang Z, Song Y, Qi H (2017) Age progression/regression by conditional adversarial autoencoder

51. Engel J, Hoffman M, Roberts A (2017) Latent constraints: learning to generate conditionally from unconditional generative models

52. Nguyen KT, Blum LC, van Deursen R, Reymond J-L (2009) Classification of organic molecules by molecular quantum numbers. ChemMedChem 4(11):1803-1805

53. Bemis GW, Murcko MA (1996) The properties of known drugs. 1. molecular frameworks. J Med Chem 39(15):2887-2893
54. Ertl P, Schuffenhauer A (2009) Estimation of synthetic accessibility score of drug-like molecules based on molecular complexity and fragment contributions. J Cheminform. 1(1):8

55. Irwin JJ, Shoichet BK (2005) ZINC - a free database of commercially available compounds for virtual screening. J Chem Inf Model 45(1):177-182

56. Polykovskiy D, Zhebrak A, Sanchez-Lengeling B, Golovanov S, Tatanov O, Belyaev S, Kurbanov R, Artamonov A, Aladinskiy V, Veselov M, Kadurin A, Johansson S, Chen H, Nikolenko S, Aspuru-Guzik A, Zhavoronkov A. MOSES GitHub repository. https://github.com/molecularsets/moses/. Accessed 15 Nov 2019

57. Preuer K, Renz P, Unterthiner T, Hochreiter S, Klambauer G (2018) Fréchet ChemNet distance: a metric for generative models for molecules in drug discovery. J Chem Inf Model 58(9):1736-1741

58. Kullback S, Leibler RA (1951) On information and sufficiency. Ann Math Stat 22(1):79-86

\section{Publisher's Note}

Springer Nature remains neutral with regard to jurisdictional claims in published maps and institutional affiliations.
Ready to submit your research? Choose BMC and benefit from:

- fast, convenient online submission

- thorough peer review by experienced researchers in your field

- rapid publication on acceptance

- support for research data, including large and complex data types

- gold Open Access which fosters wider collaboration and increased citations

- maximum visibility for your research: over $100 \mathrm{M}$ website views per year

At BMC, research is always in progress.

Learn more biomedcentral.com/submissions 